\title{
Extracting Super-resolution Structures inside a Single Molecule or Overlapped Molecules from One Blurred Image
}

Edward Y. Sheffield*, iLabY International (Div.62293017)

This article has been withdrawn by bioRxiv because it was submitted with false information. 